\title{
Empirical Green's Function Analysis of Recent Moderate Events in California
}

\author{
by S. E. Hough
}

\begin{abstract}
I use seismic data from portable digital stations and the broadband Terrascope network in southern California to investigate radiated earthquake source spectra and discuss the results in light of previous studies on both static stress drop and apparent stress. Applying the empirical Green's function (EGF) method to two sets of $M$ 4-6.1 events, I obtain deconvolved source-spectra estimates and corner frequencies. The results are consistent with an $\omega^{2}$ source model and constant Brune stress drop. However, consideration of the raw spectral shapes of the largest events provides evidence for a high-frequency decay more shallow than $\omega^{2}$. The intermediate $\left(\approx f^{-1}\right)$ slope cannot be explained plausibly with attenuation or site effects and is qualitatively consistent with a model incorporating directivity effects and a fractional stress-drop rupture process, as suggested by Haddon (1996). However, the results obtained in this study are not consistent with the model of Haddon (1996) in that the intermediate slope is not revealed with EGF analysis. This could reflect either bandwidth limitations inherent in EGF analysis or perhaps a rupture process that is not self-similar. I show that a model with an intermediate spectral decay can also reconcile the apparent discrepancy between the scaling of static stress drop and that of apparent stress drop for moderate-to-large events.
\end{abstract}

\section{Introduction}

The quest to understand earthquake source spectra, and their implications for earthquake rupture processes, dates back at least several decades. Fundamentally, an earthquake rupture will produce a (far-field) flat displacement spectrum at low frequencies, with a corner frequency that is inversely proportional to rupture length and a decay at frequencies higher than the corner. Many lines of theory and observational evidence support a high-frequency spectral decay of $f^{-2}$ (the $\omega^{2}$ model) (Brune, 1970; Hanks, 1979; Frankel, 1991; Hough and Dreger, 1995). A decay of $f^{-3}$ (or stronger) has been shown to be inconsistent with observations because it requires constant high-frequency energy with increasing moment, which is not observed (Walter $e t$ al., 1988).

However, the detailed spectral shape will reflect a myriad of rupture complexities; these have proven difficult to unravel. Several models published in the 1960s and 1970s introduced an intermediate spectral decay (Haskell, 1964; Brune, 1970) either to explain observed estimates of radiated energy (Haskell, 1964) or to allow for partial stress-drop ruptures (Brune, 1970). More recently, self-healing slippulse models have been proposed based on a wealth of detailed source models resulting from the inversion of strongmotion data (Heaton, 1990). The primary feature of a slip-pulse model is that, at any one time during the rupture of a large event, slip is occuring only in a narrow band that propagates along a fault. The overall rupture dimension of a large earthquake is thus much larger than the instantaneous extent of the slip pulse.

It was recently shown that a simple dynamic rupture model, incorporating the effects of directivity, will produce rms-average spectral levels that are amplified with respect to a one-corner Brune model (Haddon, 1996). For the acceleration spectrum, a slope of -0.5 is found for a complete stress-drop model, while a slope of approximately -1 can be obtained for a fractional stress-drop rupture such as the abruptly healing slip-pulse model. Deviations from the simple $\omega^{2}$ model can also be shown to be a consequence of finite-fault effects for large events. As shown by Atkinson and Silva (1997) and Beresnev and Atkinson (1999), a propagation of Brune point sources along a fault plane will result in an apparent sag of spectral amplitudes, relative to the omega-square model, at intermediate frequencies.

While the aforementioned efforts toward understanding earthquake rupture processes have continued, parallel efforts have progressed on the understanding of observational earthquake spectra, utilizing the increasing data base of strong motion and broadband waveform recordings. While progress has been made along several lines, one key paradox has emerged. Empirical Green's function (EGF) results have 
consistently argued for fairly simple source spectra of magnitude 2-7 events, with constant Brune stress drop, $\sigma_{\mathrm{b}}$, estimated from the corner frequency (e.g., Frankel et al., 1986; Mori and Frankel, 1990; Abercrombie and Leary, 1993; Frankel, 1995; Hough and Dreger, 1995). Brune stress drop provides an estimate of the dynamic stress drop that can be related, with assumptions regarding a rupture model, to static stress drop. Often, $\sigma_{\mathrm{b}}$ and static stress drop are used interchangeably, with the former assumed to represent the latter.

Direct static stress drop estimates, as inferred from finite-fault models of larger events, are also generally found to not scale with moment and to be in the range 1-10 MPa (e.g., Humphrey and Anderson, 1995; Hough, 1996). However, investigations of radiated energy have consistently argued for complex spectral shapes and/or apparent stress drop that increases with moment (e.g., Smith et al., 1991; Kanamori et al., 1993; Mayeda and Walter, 1996; Atkinson and Silva, 1997). An intermediate spectral decay has also been inferred using teleseismic data (e.g., Boatwright and Choy, 1992). It has been suggested that one resolution of this paradox could involve the slip-pulse model for rupture (Mayeda and Walter, 1996). The model of Haddon (1996) suggests that the high values of radiated energy (implying that spectral shapes are elevated compared to an $\omega^{2}$ model) of recent moderate-to-large California earthquakes may in part reflect an incomplete accounting of directivity effects, although his results predict that deconvolved (EGF) source spectra should also be characterized by an intermediate (and azimuthdependent) decay.

To further investigate this issue, I use broadband recordings of the 1992 Joshua Tree, California, earthquake sequence from the Terrascope network in southern California (Hauksson et al., 1993) and of moderate events from the Landers, California, sequence of 1992 (Fig. 1) (Hough, 1994). The Joshua Tree earthquake sequence is attractive because it has already been analyzed with an EGF approach (Hough and Dreger, 1995) and radiated energy approaches (Kanamori et al., 1993; Mayeda and Walter, 1996). A static stress drop estimate of $\approx 5 \mathrm{MPa}$ is inferred from the mainshock-source model, and Brune stress drop estimates of the mainshock and aftershocks support a constant scaling over the magnitude range 2.5-6. However, Kanamori et al., (1993) and Mayeda and Walter (1996) find high apparent stress for the mainshock ( $\approx 20-30 \mathrm{MPa})$ and evidence for a complex source spectral shape.

\section{Data}

To identify suitable EGF event pairs or clusters, I first apply a simple pair-finding algorithm using all $M 3.8$ and greater events since 1991, using the Southern California Seismic Network (SCSN) catalog. In general, suitable EGF events for local events are within $1 \mathrm{~km}$ of each other; regional studies have successfully used EGF events separated by distances of a few kilometers (Dreger, 1994; Velasco et al., 1994; Jones and Hough, 1995). I first search for events with an epicentral distance within $2 \mathrm{~km}$ and a magnitude difference of 0.7 units or more; these criteria are crude, but a subsequent waveform evaluation is made to confirm potential event pairs. To be a suitable EGF event, an earthquake must exhibit a high degree of waveform similarity to its mainshock and be enough smaller than its mainshock to have a distinctly different corner frequency, and yet still be large enough to provide adequate long-period signal-to-noise levels to resolve source properties for the larger event. Suitable pairs are thus typically within 1-2 magnitude units of each other. For each mainshock, the entire catalog is searched to find potential EGF events.

Because the goal is to investigate moderate-to-large ( $M$ 4-7) earthquakes, the ideal EGF clusters contain a mainshock of magnitude 5.5-7+. Including aftershocks, 10 events in this magnitude range were recorded between January 1991 and June 1996. The most notable of these events are the 23 April $1992 M 6.1$ Joshua Tree and 28 June 1992 M 7.3 Landers earthquakes (Hauksson et al., 1993). The 28 June $1992 M$ 6.5 Big Bear earthquake (Jones and Hough, 1995), the 17 January $1994 M 6.7$ Northridge earthquake (Jones et al., 1994), and the 20 September 1995 M 5.8 Ridgecrest, California event (Hauksson et al., 1995). The pair-finding algorithm reveals a paucity of candidate EGF events for the Landers earthquakes; there are only four events, the largest of which is $M$ 4.4. Similarly, only a single candidate EGF event is found for the Northridge event, also of relatively small magnitude ( $M$ 3.9). This may reflect a general tendency for the bigger earthquakes to leave insufficient residual stress to generate substantial aftershocks that are close to the mainshock hypocenter. The Ridgecrest sequence contains several candidate EGF events; however, upon closer inspection, none exhibit the required degree of waveform or spectral similarity. The sequence also occurred at the edge of the Terrascope network and so is relatively poorly recorded.

Of the available data, two clusters are best suited for analysis: the Joshua Tree earthquake, its foreshock, and several of its aftershocks; and a $M 5.6$ early aftershock of the Landers event that occurred 3 min after the mainshock and was associated with mapped surface rupture on the Eureka Peak fault (the Yucca Valley event) (Hough et al., 1993; Hough, 1994). The source dimension of the mainshock in both clusters is well constrained, in the former case from the finite-fault inversion and in the latter case from the densearray analysis of strong-motion recordings (Hough et al., 1993).

The Joshua Tree sequence was well recorded by the broadband Terrascope network (Kanamori et al., 1991), but broadband recordings of the Yucca Valley event are complicated by the substantial coda of the Landers mainshock. For this event, I use data from a portable instrument deployment (GEOS instruments) (Borcherdt et al., 1985) that recorded the Landers mainshock and much of the early aftershock sequence (Hough et al., 1993). The GEOS instruments that recorded the Yucca Valley event were deployed in a 
dense-array configuration. Because the information is redundant (for gross characterization of source and attenuation properties), I analyze data from a single station, MVB. Figure 1 and Table 1 present locations and magnitudes of events analyzed.

For the Joshua Tree mainshock, low-gain force-balance accelerometer (fba) recordings are available from Terrascope stations PFO and GSC; very broadband (vbb) Streckheisen recordings are used for the mainshock at other stations and all smaller events. The fba data are sampled at 80 samples per sec; the vbb channels at 20 samples per sec. The vbb instrument response is flat to the velocity over the frequency band $\approx 0.0027-7 \mathrm{~Hz}$; for the Joshua Tree data, I generally find a usable bandwidth of $0.07-6 \mathrm{~Hz}$.

For the GEOS data from station MVB, I use fba record- ings and obtain a bandwidth of roughly $0.4-30 \mathrm{~Hz}$. In the subsequent analysis, fba data are integrated to the velocity and high-pass filtered using a third-order butterworth filter and a corner of $0.05 \mathrm{~Hz}$ for the Joshua Tree events (Fig. 2a) and $0.1 \mathrm{~Hz}$ for the Yucca Valley data (Fig. 2b).

\section{Analysis}

\section{EGF Analysis}

Using velocity data, I window the primary shear-wave arrivals using window lengths that vary by event-station pair but are consistent within a given event cluster at a given station. Spectra are estimated from both horizontal components using a cosine taper and a standard fast Fourier trans-

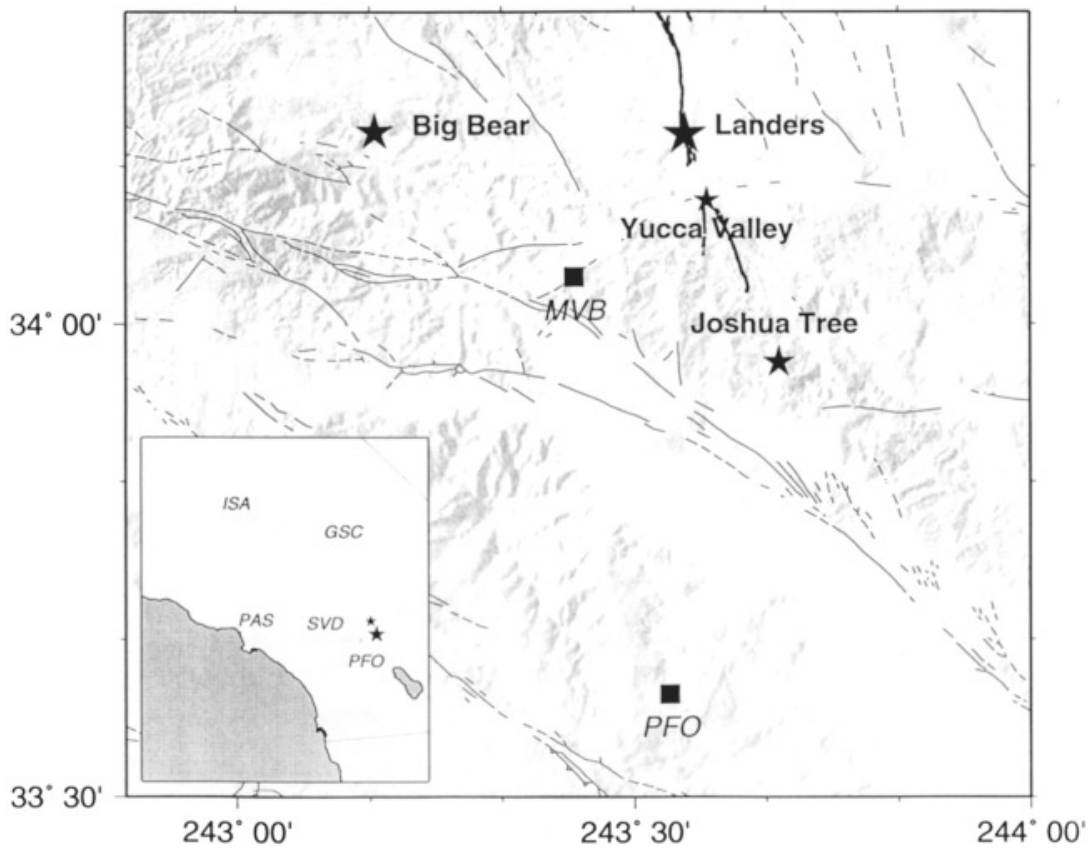

Figure 1. Events, stations, and regional setting of data analyzed. Large stars indicate $M$ 7.3 Landers and $M$ 6.5 Big Bear earthquakes of 28 June 1992; smaller stars indicate the $M$ 5.6 Yucca Valley aftershock of 28 June 1992 and and the $M 6.1$ Joshua Tree event of 23 April 1992. Small squares indicate the portable station MVB and Terrascope station PFO, which provided the data analyzed in this study. Inset shows regional setting and locations of Terrascope stations PFO, PAS, SVD, GSC, and ISA (Joshua Tree and Yucca Valley epicenters are replotted with stars). Light lines indicate regional faults; dark lines indicates southern extent of surface rupture of the 1992 Landers earthquake, for reference. Shading indicates topographic relief.

Table 1

Events Analyzed

\begin{tabular}{|c|c|c|c|c|c|c|c|c|}
\hline Month & Day & Hour (UTC) & Latitude & Longitude & $M$ & $f_{c}(\mathrm{~Hz})$ & sdev & $\sigma$ \\
\hline 04 & 23 & 0225 & 33.9562 & -116.3173 & 4.4 & 1.71 & 0.6 & 4.4 \\
\hline 04 & 23 & 0450 & 33.9612 & -116.3175 & 6.1 & 0.17 & 0.08 & 1.4 \\
\hline 04 & 23 & 0510 & 33.9577 & -116.3297 & 4.4 & 0.50 & & 0.1 \\
\hline 05 & 04 & 1619 & 33.9410 & -116.3040 & 4.8 & 0.84 & 0.45 & 4.2 \\
\hline 05 & 18 & 1544 & 33.9510 & -116.3373 & 5.0 & 0.69 & & 0.8 \\
\hline 05 & 06 & 0238 & 33.9426 & -116.3143 & 4.7 & 0.92 & & 2.7 \\
\hline 06 & 28 & 1200 & 34.1307 & -116.4080 & 5.6 & 0.64 & & 16.4 \\
\hline 06 & 30 & 0006 & 34.1248 & -116.4012 & 4.4 & 2.06 & & 8.7 \\
\hline 07 & 05 & 1352 & 34.1208 & -116.3913 & 3.9 & 2.84 & & 4.1 \\
\hline 07 & 10 & 0241 & 34.1200 & -116.3942 & 4.0 & 2.84 & & 5.7 \\
\hline
\end{tabular}

Month, day, hour, and minute of event. Magnitudes are from SCSN catalog; Joshua Tree magnitude is moment magnitude, $\mathbf{M}_{w} . f_{\mathrm{c}}$ and $\sigma$ are corner frequencies and stress drops (in MPa) determined from $S$ waves, and sdev gives sample standard deviation for events for which at least four individual $f_{c}$ estimates are made. First group represents the Joshua Tree events analyzed; second group gives Yucca Valley events. Joshua Tree mainshock corner frequency represents average value estimated from only the four $M>4$ EGF events. 
(a)

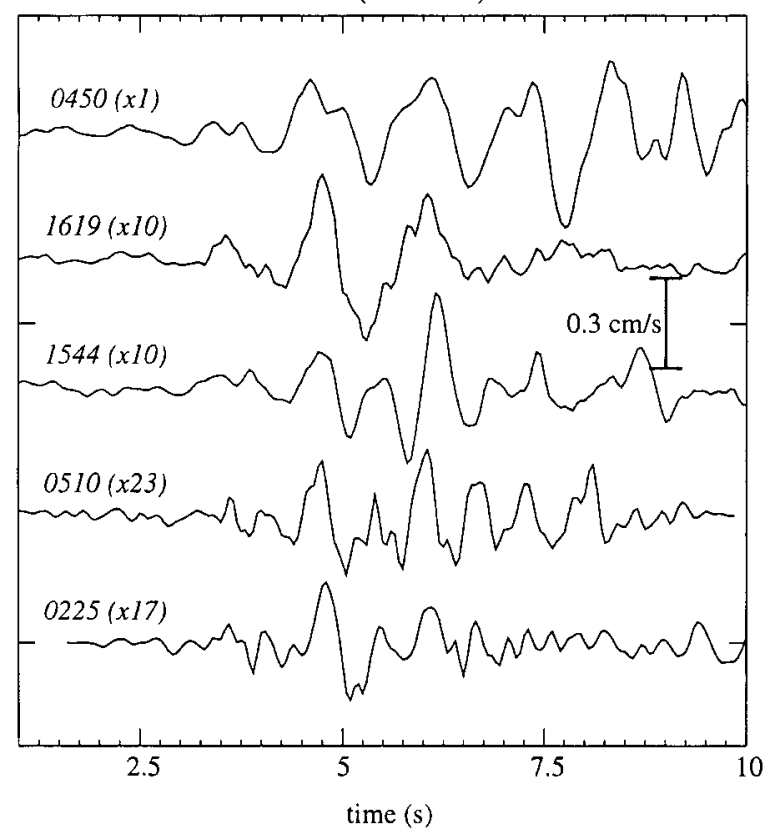

(b)

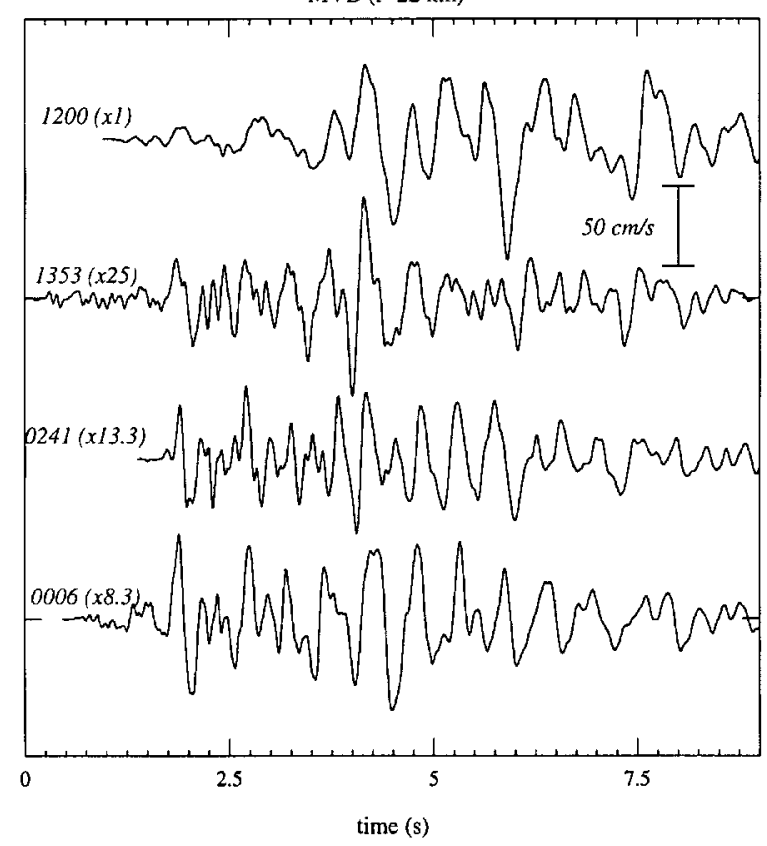

Figure 2. (a) Scaled velocity traces for the north component of the Joshua Tree mainshock (top) and four events used as empirical Green's functions, recorded at station PAS. Events are identified by hour and minute (e.g., 0450 = 04:50 UTC) (see Table 1). Records are scaled and offset to illustrate waveform similarity. Multiplicative scaling factor shown for each of the EGF events. Spectra are calculated using the entire (windowed) time series shown. (b) Scaled velocity traces for the $M 5.6$ Yucca Valley event (top) and the three events used as empirical Green's functions, recorded at the portable GEOS station MVB. Multiplicative scaling factor shown for each of the EGF events. Spectra are again calculated using the entire (windowed) time series shown. form (FFT); with boxcar smoothing over $\pm 0.1 \mathrm{~Hz}$. Deconvolution is then performed in the frequency domain, via a straightforward process of spectral division. Following Hough (1997), I then compute a root mean square (rms) average of the spectra from the two horizontal components (Fig. 3a). Superimposed on the deconvolved spectra are ratios of theoretical $\omega^{2}$ spectra that best fit the logarithm of the calculated spectra (in a least-squares sense). The regression is done using a grid-search method to find the pair of cornerfrequency values that gives the optimal least-squares misfit. Each deconvolved source spectrum thus yields a cornerfrequency estimate for the mainshock and the EGF event. For each mainshock, a separate corner-frequency estimate is obtained for each EGF event, for each station. For the EGF events, one corner-frequency value is obtained at each station (that recorded that event).

All corner-frequency values are expected to reflect directivity effects. A finite-fault inversion of the Joshua Tree mainshock (Hough and Dreger, 1995) provides a more thorough accounting of these directivity effects, and so is expected to provide better resolution of source size/stress drop. For the Joshua Tree event, I first average all results from the deconvolutions using different EGF events to obtain one mainshock corner-frequency estimate for each site, then average (arithmetically) those results over all sites. Table 2 illustrates the variability of $f_{\mathrm{c}}$ estimates, for both the mainshock and the EGF events, obtained at the different stations. Final averaged values for all the events analyzed are summarized in Table 1. As discussed by Hough (1997), the consistency of $f_{\mathrm{c}}$ estimates obtained using different EGF events provides a good estimate of the uncertainties. Table 1 includes the sample standard deviations (sdev) for those $f_{c}$ values for which at least four individual $f_{c}$ estimates are obtained. I note that the sdev values for the mainshock are likely to be overestimates because they include directivity effects (Hough and Dreger, 1995). Estimating an sdev for the mainshock from stations PAS and PFO, I obtain values of 0.03 and $0.04 \mathrm{~Hz}$, respectively.

Figure 3a supports the conclusions of a previous EGF study of the Joshua Tree event (Hough and Dreger, 1995), that the deconvolved source spectra do not reveal significant departure from an $\omega^{2}$ model and that the inferred Brune stress-drop results are quite constant with moment (Table 1). Figure $3 \mathrm{~b}$ reveals that the $\omega^{2}$ model provides a good fit to the deconvolved source spectra for the Yucca Valley event, with Brune stress-drop values ranging from 4.1 to $16.4 \mathrm{MPa}$.

The ratios of high- to low-frequency deconvolved source spectra levels can also be estimated. The high-frequency level predicted for a constant stress drop, omegasquare model (given the observed low-frequency level) is illustrated for the Yucca Valley events (Fig. 3b). Generally, good consistency is found with the predictions.

The mainshock corner frequencies/stress-drop values can also be interpreted in terms of overall rupture dimension (Brune, 1970). Assuming a source-time function half-width given by $t_{1 / 2}=0.37 / f_{c}$ (Brune et al., 1979) where the rupture 

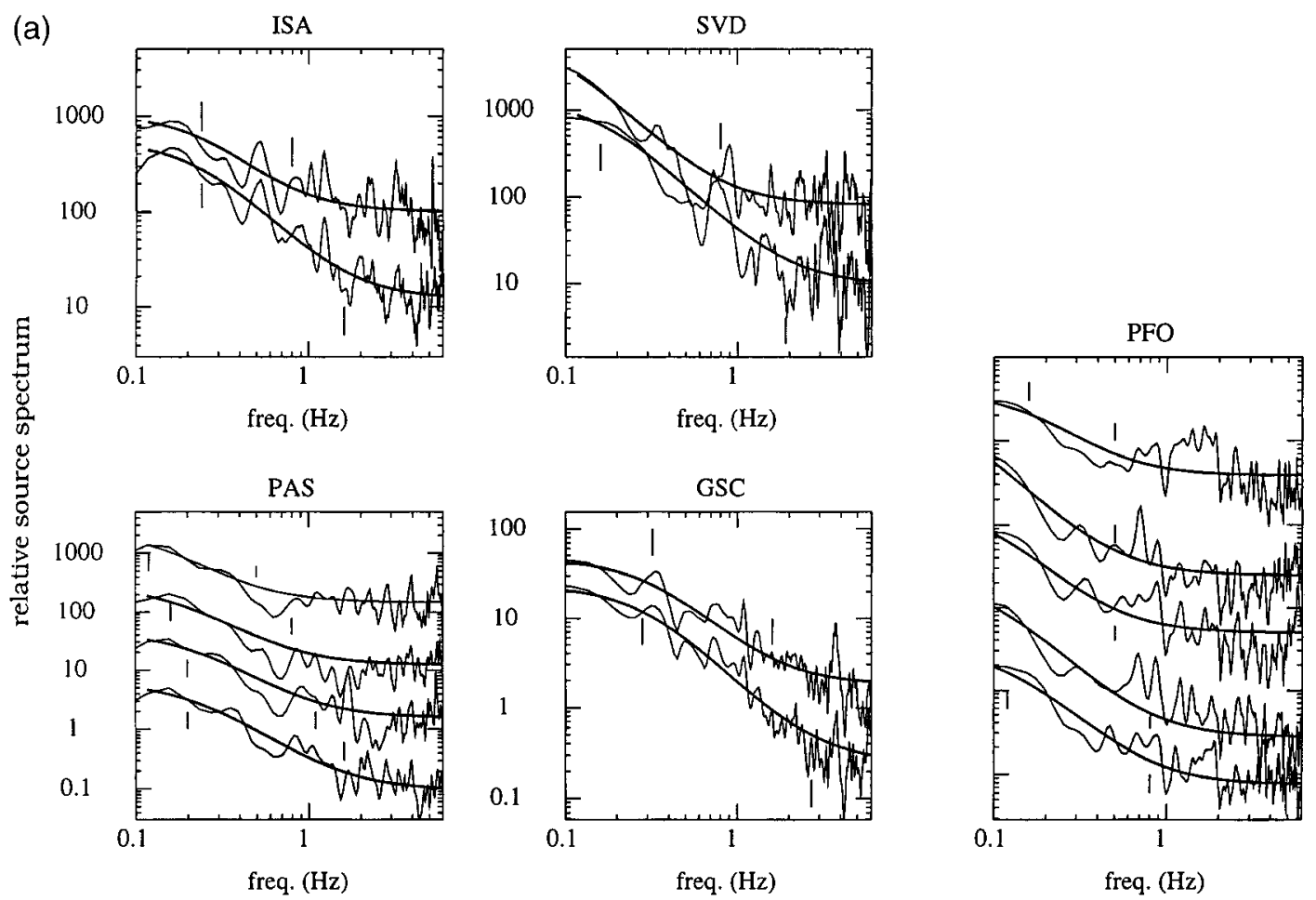

(b)

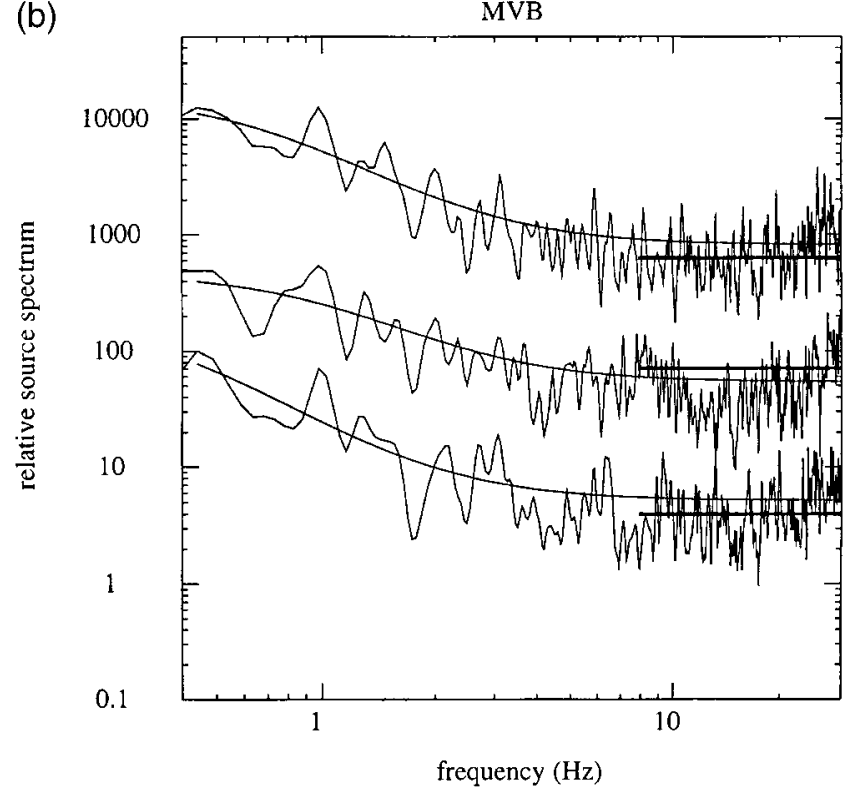

velocity is taken to be $0.9 \beta$; a $0.17 \mathrm{~Hz}$ corner frequency for the Joshua Tree event implies an overall rupture dimension of $14.3 \mathrm{~km}$, just slightly larger than the $10-12-\mathrm{km}$ diameter obtained via the finite-fault inversion. The corner frequency for the Yucca Valley event is less well constrained, with estimates from the three EGF deconvolutions at station MVB yielding a range of $0.32-0.96 \mathrm{~Hz}$, implying a rupture dimension range of $2.4-7.3 \mathrm{~km}$. This is smaller than the previously inferred 11-km dimension (Hough et al., 1993). As I discuss in a later section, it is possible that the corner-
Figure 3. (a) The rms horizontal deconvolved (relative) source spectra are shown for five Terrascope stations that recorded the $M 6.11992$ Joshua Tree, California, earthquake, using up to five smaller nearby events as empirical Green's functions (EGF events used are identified by hour and minute). Superimposed on each source spectrum is the best-fitting theoretical model of $\omega^{2}$ source spectra; a regression determines the optimal mainshock and empirical Green's function corner frequency for each pair. Deconvolved source spectra are offset for clarity. Small tick marks show corner-frequency estimates (those within the range 0.1-30 Hz) (see Table 2). (b) Same (a) for Yucca Valley event. Three smaller events are used as empirical Green's functions (Fig. 2b) (Table 1). Solid lines indicate highfrequency spectral levels consistent with constant stressdrop scaling, relative to observed high-frequency levels.

frequency estimates for this event are biased toward high values.

While the EGF results are thus generally consistent with a one-corner model and a Brune stress drop comparable to static stress drop, it is illustrative to return to the observed (not deconvolved) velocity spectra. One corner frequencies are constrained, it is possible to use colocated event clusters to invert spectra for a common frequency-independent attenuation parameter and site response (Hough, 1997). In this multiple-empirical Green's function (MEGF) approach, the 
Table 2

EGF Results for Joshua Tree Mainshock

\begin{tabular}{ccccc}
\hline Station & EGF Event & $f_{\mathrm{c} 1}(\mathrm{~Hz})$ & $f_{\mathrm{c} 2}(\mathrm{~Hz})$ & $\kappa(\mathrm{sec})$ \\
\hline ISA & 0225 & 0.24 & 1.6 & 0.079 \\
& 1619 & 0.24 & 0.8 & \\
PAS & 0225 & 0.20 & 1.6 & 0.099 \\
& 0238 & 0.20 & 1.1 & \\
& 1544 & 0.16 & 0.8 & \\
& 1619 & 0.12 & 0.5 & \\
GSC & 0225 & 0.28 & 2.7 & 0.103 \\
& 1619 & 0.32 & 1.6 & \\
SVD & 0225 & 0.16 & 1.9 & -0.090 \\
& 1544 & 0.08 & 0.8 & \\
PFO & 0225 & 0.12 & 0.8 & -0.070 \\
& 0238 & 0.08 & 0.8 & \\
& 0510 & 0.08 & 0.5 & \\
& 1544 & 0.04 & 0.5 & \\
& 1619 & 0.16 & 0.5 & \\
\hline
\end{tabular}

Terrascope station hour and minute of EGF event (see Table 2), corner frequency estimate for mainshock, and EGF event, respectively, and attenuation parameter $\kappa$.

attenuation parameter and the site response are constrained by spectra from multiple events with a range of magnitudes. The usual trade-offs between corner frequency and attenuation are also avoided because the source parameters have been determined by the early EGF analysis. The combined source, path, and site terms should provide an adequate characterization of the spectral shape (Hough, 1997; Hough et al., 1999).

However, applying MEGF analysis to the Joshua Tree clusters yields negative attenuation parameters at several of the stations. Figure 4, which shows the mainshock spectra at stations PAS, GSC, and PFO, illustrates why the overall spectral shapes at PFO especially, are not well fit by an $\omega^{2}$ model. It is illuminating to focus on the data from PFO, which is the closest Terrascope station to the Joshua Tree events and is expected to be relatively free of strong site effects (Berger et al., 1984; Fletcher et al., 1990; Aster and Shearer, 1991; Kato et al., 1995). Previous studies (e.g., Hough and Anderson, 1988) have also provided a characterization of local and regional attenuation at PFO. Superimposed on the PFO mainshock spectrum in Figure 4 is an $\omega^{2}$ source (velocity) model, with the corner frequency determined from the EGF analysis, assuming no anelastic attenuation. The shallow decay of the spectrum relative to the $\omega^{2}$ model gives rise to a negative attenuation parameter (and $Q$ value). These results will be discussed at length in a later section.

The raw spectra from the Yucca Valley sequence do exhibit a decay stronger than $\omega^{2}$, and so one is able to analyze these events to investigate the consistency of their radiated spectra with the $\omega^{2}$ model. Applying the MEGF method with corner frequencies for all events constrained according to the prior EGF analysis, the spectra are fit by

$$
A(f, r)=A_{0}(f) \mathrm{e}^{-\pi \kappa f},
$$

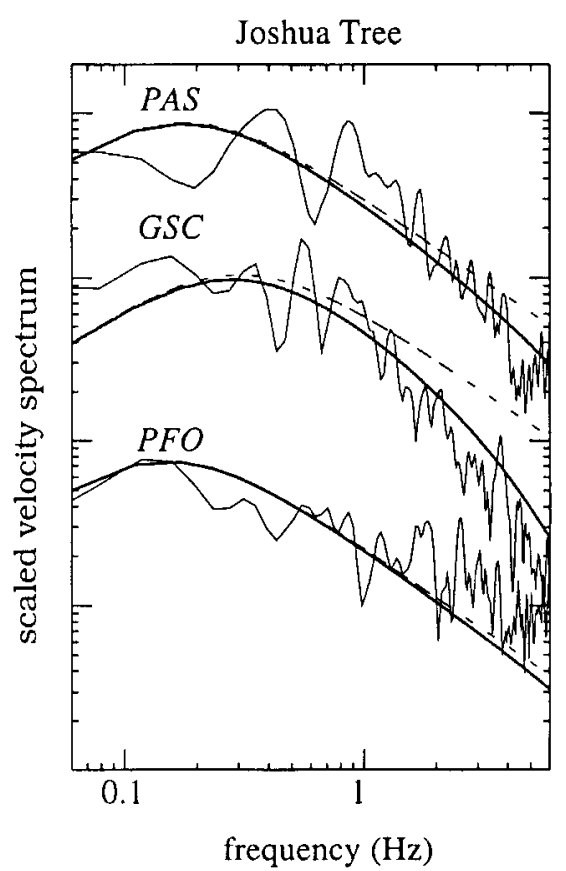

Figure 4. Scaled, offset rms velocity spectra for the Joshua Tree mainshock at stations PFO (bottom), GSC (middle), and PAS (top). Superimposed are $\omega^{2}$ (velocity) source models (dashed lines), with the corner frequency determined from an average of the results from that station and a low-frequency spectral level chosen to roughly fit the data, as well as the same models with an appropriate attenuation parameter added (solid lines).

where $A_{0}(f)$ is the source spectrum and the attenuation parameter, $\kappa$, is equated to the path-dependent attenuation term (Anderson and Hough, 1984),

$$
\kappa=\int \frac{d r}{Q_{i} \beta},
$$

where $Q_{i}$ is a frequency-independent attenuation parameter and $\beta$ is the shear-wave velocity. $A_{0}(f)$ is given by the $\omega^{2}$ model (for velocity)

$$
A_{0}(f)=\frac{\alpha_{0} f}{1+\left(f / f_{\mathrm{c}}\right)^{2}}
$$

where $f_{\mathrm{c}}$ is the corner frequency and the high-frequency spectral decay is assumed to be -2 . If the spectra are well characterized by exponential decay, then the slope of the decay can be interpreted using equation (2) for the frequency-independent component of attenuation (Anderson and Hough, 1984). This leaves open the possibility that a total $Q^{-1}$ will be given by

$$
1 / Q_{i}+1 / Q_{f}
$$


where the frequency-dependent term $Q_{f}$ is linearly dependent on frequency (see Hough [1997] for further discussion). Determination of this component requires consideration of absolute spectral amplitudes, which is not possible with the single-station method employed here. The absolute amplitude term, $\alpha_{o}$, in equation (3) will thus incorporate change in the overall spectral level due to either geometrical spreading or a component of attenuation that is proportional to frequency, as well as the inherent source amplitude. The attenuation estimates that are inferred from the frequencyindependent component can thus be considered lower bounds.

The observed velocity spectra are first corrected for the $\omega^{2}$ shape (using constrained corner frequency); the lowfrequency asymptote and spectral decay then determine $\alpha_{\mathrm{o}}$ and $\kappa$. Again, this analysis resolves only $Q_{f}$, from the inferred $\kappa$ values. The resulting spectral fits, and residuals (averaged from all events), are shown in Figure 5. Although at first glance this model appears to provide a good characterization of the observed spectra, closer inspection reveals a significant mismatch to the spectra at the lowest frequencies, $0.5-0.8 \mathrm{~Hz}$ (a range for which good signal-to-noise levels exist).

\section{Observations at $\mathrm{PFO}$}

The spectra shown in Figures 4 and 5 will, as noted, reflect a combination of source, path (attenuation, scattering, and geometrical spreading), and site effects. Attenuation is expected to affect the spectra according to $\left(\mathrm{e}^{[-\pi f \delta d r / Q \beta]}\right)$ where $Q$ is now the total quality factor. Numerous studies have shown that $Q=Q_{0} f^{a}$ where $a$ is between 0 and 1 (e.g., Singh and Herrmann, 1983; Aki, 1991; Raoof et al., 1999). A total $Q$ of the form given by equation (4) can be modeled by this functional form over a finite-frequency band (Anderson and Hough, 1984). With an attenuation function of this form, the rate of high-frequency spectral decay can only be increased. That is, the overall exponential factor will inevitably decrease with increasing frequency. In fact, previous studies provide constraint on attenuation at $\mathrm{PFO}$, with $Q$ values of $\approx 250$ at $5 \mathrm{~Hz}$ and $\approx 500$ at $20 \mathrm{~Hz}$ (Hough and Anderson, 1988). Thus, attenuation cannot be invoked to explain the observed discrepancies at PFO.

It is also difficult to appeal to site response to explain anomalously high spectral levels at high frequencies, particularly at station PFO. Station PFO is located at the Pinyon Flat Observatory within the Southern California batholith, away from topographic variation. This site has been chosen for numerous geophysical/seismological investigations based on its expected minimal site complexity (e.g., Berger et al., 1984).

Several previous studies have specifically investigated site response at and near PFO, using data from the broadband Anza array (Vernon, 1989; Aster and Shearer, 1991). Borehole $S$-wave velocity results have been determined for the upper $300 \mathrm{~m}$ for PFO (Fletcher et al., 1990), and site response has been calculated using borehole seismometers at Anza
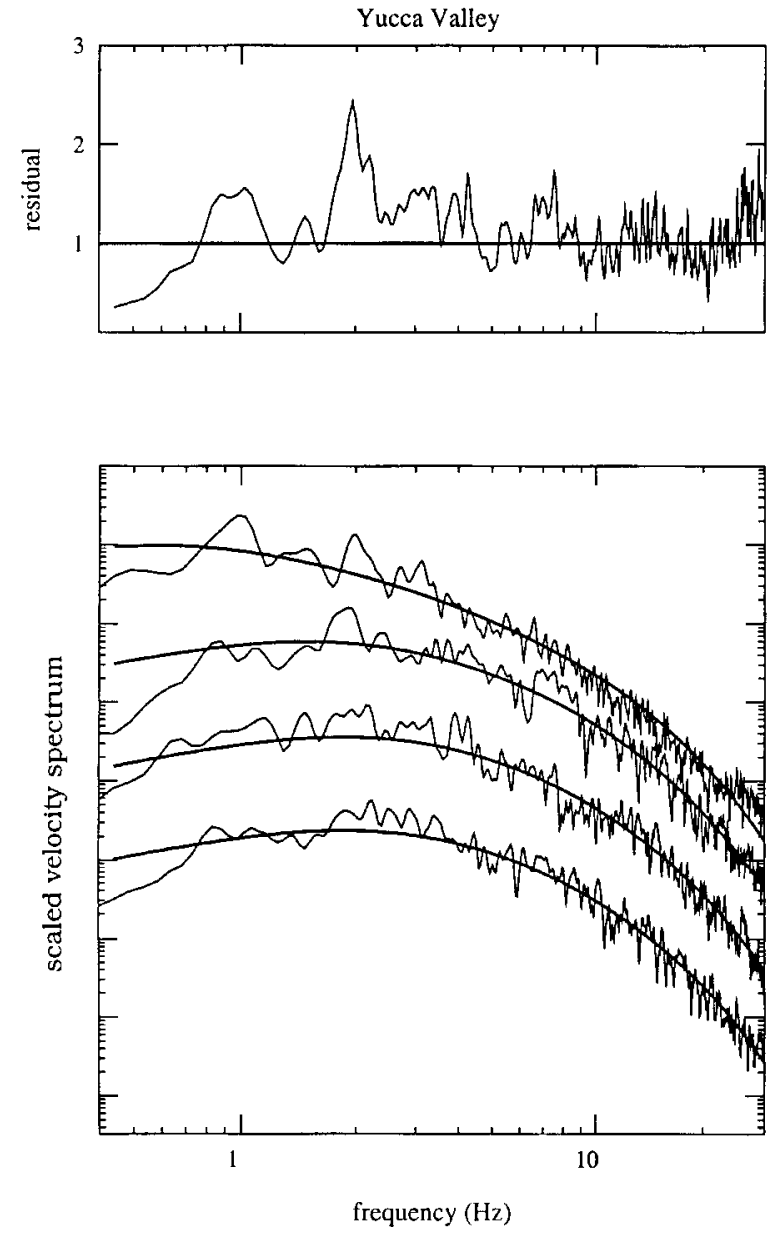

Figure 5. Scaled, offset rms average velocity spectra at station MVB for the $M 5.6$ Yucca Valley event and three $M$ 3.9-4.4 events from its aftershock subsequence (bottom panel). The top spectrum corresponds to the Yucca Valley event; the bottom three spectra are offset for clarity (all three are roughly of comparable amplitude). The top panel shows the residual between the model and the raw spectra, averaged over the four events.

station KNW (Aster and Shearer, 1991), which is roughly comparable to PFO in the extent of the near-surface weathered layer and near-site geology (Vernon, 1989). The observed response of the upper $300 \mathrm{~m}$ at $\mathrm{KNW}$ are shown to be well modeled by the theoretical one-dimensional response of the borehole-velocity model (Aster and Shearer, 1991).

The Fletcher et al., (1990) velocity model (the upper five layers shown in Table 3, with the basement extended to essentially form a half-space) yields the theoretical one-dimensional site response shown in Figure 6. Perhaps surprisingly given the hard rock nature of the near-surface geology at PFO, this response does reveal a significant amplification at higher frequencies, up to a factor of 5-6 over a broadfrequency band, roughly 5-25 Hz. However, correcting the observed Joshua Tree spectrum at PFO by this site-response estimation (Fig. 7) does not result in appreciable changes in 
Table 3

Model

\begin{tabular}{rcccc}
\hline$Z(\mathrm{~m})$ & $\alpha(\mathrm{m} / \mathrm{sec})$ & $\beta(\mathrm{m} / \mathrm{sec})$ & $\rho\left(\mathrm{kg} / \mathrm{m}^{3}\right)$ & $Q$ \\
\hline 3 & 400 & 200 & 1.5 & 7 \\
2 & 700 & 400 & 2.7 & 7 \\
10 & 1700 & 900 & 2.7 & 7 \\
35 & 3000 & 1650 & 2.7 & 7 \\
15 & 3100 & 1650 & 2.7 & 7 \\
235 & 5300 & 3000 & 2.7 & 50 \\
1400 & 5300 & 3000 & 2.7 & 500 \\
2000 & 5400 & 3100 & 2.7 & 999 \\
\hline
\end{tabular}

Thickness of layer (Z), $P$ - and $S$-wave velocities ( $\alpha$ and $\beta$ ), density $(\rho)$, and quality factor $(Q)$. The $Q$ value for the first layer is determined from observed spectral shapes; $Q$ values are assumed for the deeper layers. These $Q$ values will trade off to some extent, as discussed in the text.

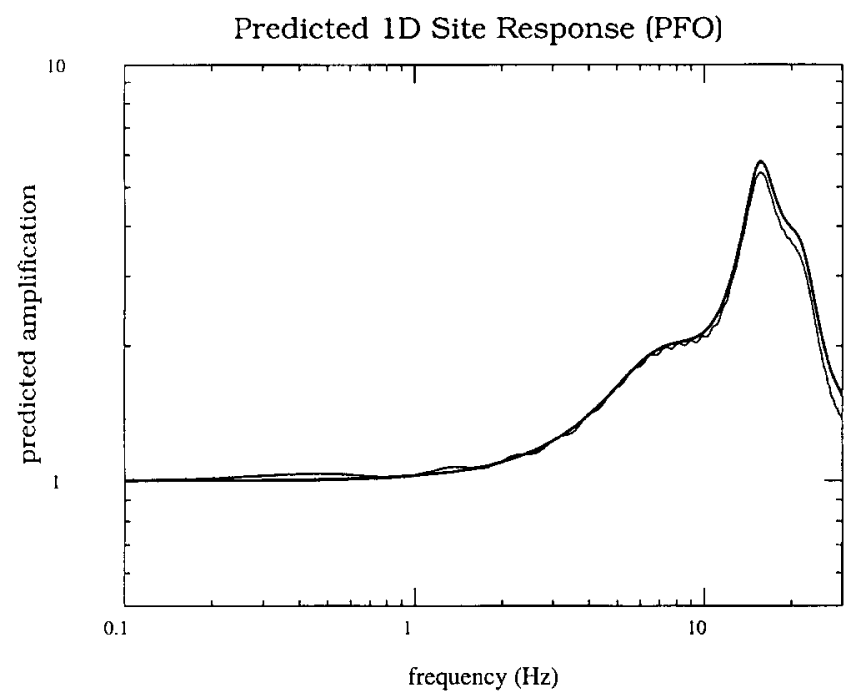

Figure 6. Theoretical one-dimensional response calculated using the velocity model shown in Table 3 for station PFO. The light line corresponds to the response of the upper $50-70 \mathrm{~m}$, for which $S$-wave velocities are constrained from borehole studies (Fletcher et al., 1990). The dark line corresponds to the full model, which is extended to match a regional velocity model for the Anza area. The close correspondence of the two curves indicates the primary control of site response by the upper $70 \mathrm{~m}$.

spectral shape at frequencies below 3-5 Hz; the observed spectra are still not well matched by a single-corner model (with the corner frequency fixed to a value determined by EGF analysis).

It is straightforward to estimate the frequency band over which site effects will be important, given the thickness of the low-velocity layer and its vertical extent. A single lowvelocity layer will give rise to a fundamental resonance whose frequency is given by $\beta / 4 h$ where $h$ is the thickness of the layer; higher overtones will occur at multiples of this frequency. The effect of multiple layers will be more complex, depending on the nature of the velocity structure (Ken-

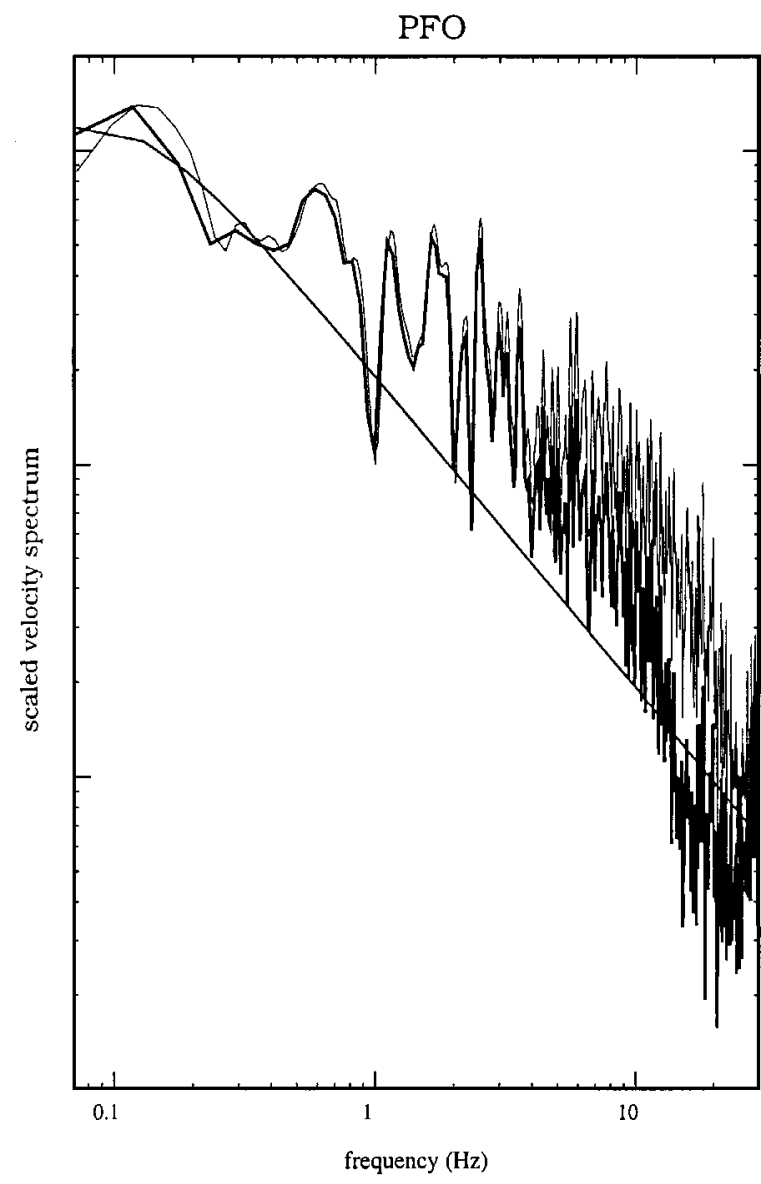

Figure 7. The (scaled) Joshua Tree velocity spectrum at PFO plotted from Figure 3 (light line), along with the spectrum corrected for the (deeper model) site response shown in Figure 6 (dark line). The theoretical $\omega^{2}$ source model is also replotted; the corrected spectrum is still well above this curve over the frequency range $\approx 1-10 \mathrm{~Hz}$.

nett and Kerry, 1979). However, the same basic relationship will govern the dependence of the dominant frequency on layer thickness. Thus, the 15-m-thick low-velocity (weathered) layer at PFO (the most conspicuous low-velocity zone) will give rise to dominant amplifications at frequencies of roughly $10 \mathrm{~Hz}$ and above. If one considers the expected response of the combined low-velocity unit (roughly $1300 \mathrm{~m} /$ $\mathrm{sec}$, on average) above $50 \mathrm{~m}$, one obtains a quarter-wavelength resonance at roughly $6.5 \mathrm{~Hz}$. The estimated onedimensional response shown in Figure 6 appears to represent a combination of these two effects.

To obtain significant site response at lower frequencies than those caused by the upper $50-70 \mathrm{~m}$ (that is, lower than $6 \mathrm{~Hz}$ ) requires a much deeper layer, with suitably low velocity. For example, a layer with a 500-m depth extent would require a $\beta$ of $2000 \mathrm{~m} / \mathrm{sec}$ to give rise to a $1-\mathrm{Hz}$ resonance (with an underlying impedance contrast sufficient to cause amplifications). However, Fletcher et al. (1990) estimated a $\beta$ value of $2800 \mathrm{~m} / \mathrm{sec}$, for depths of $50 \mathrm{~m}$ and below. 
To investigate possible effects of a deeper velocity structure, I extrapolate the Fletcher et al. (1990) velocity model to match deeper values of $\beta$ estimated from the $P$ wave velocity model of Scott et al., (1994) and recompute the one-dimensional site response. Figure 6 reveals that the predicted response from this model differs only slightly from that estimated from the more shallow model, confirming that the upper $50 \mathrm{~m}$ contributes the vast majority of the site response observed at PFO.

\section{Interpretation}

EGF Versus (Uncorrected) Spectral Results

Although the Joshua Tree recording at PFO is a single observation, it was chosen for in-depth investigation because it provides an illustration of the general paradox (i.e., low Brune stress drop versus high apparent stress drop). It was also chosen for investigation because, unlike other recordings, available previous information on velocity and attenuation structure allows one to sort out the effects of different factors that will shape the high-frequency spectrum. This recording illustrates why high apparent stress drop and low Brune/static stress drop values were obtained for this event. That is, while EGF analysis (the method used by Hough and Dreger [1995]) reveals a corner frequency that implies a typically low Brune stress drop (56 bar), the observed spectrum exhibits a decay more gradual than $\omega^{2}$, which would imply high-radiated energy (and apparent stress drop) compared to that predicted by the $\omega^{2}$ model.

The results obtained in this study, at PFO and elsewhere, are generally consistent with certain aspects of the model presented by Haddon (1996). This model, which incorporates both directivity and a model with self-similar rupture healing, predicts a spectral decay elevated with respect to a standard $\omega^{2}$ model.

However, the Haddon (1996) model also predicts that, on average, EGF analysis will yield deconvolved source spectra with $\omega^{-1}$ decay above the mainshock corner. This does not appear consistent with the results for the Joshua Tree mainshock analyzed using the $M 4.4-5$ aftershocks as Green's function events. One is left with two possible explanations: (1) that EGF deconvolutions are unable to resolve the full character of the spectrum given the inevitable bandwidth limitations, namely, given the requirement that EGF events contain sufficient long-period energy, one is typically restricted to using events that are only one, or at most two, magnitude units smaller than the mainshock, or (2) the source process that gives rise to the intermediate decay is not self-similar in its scaling, so its spectral signature is not revealed with EGF deconvolution. For example, if the scaling of an intermediate spectral decay was controlled by the slip-pulse length and this length was not a strong function of moment, the deconvolutions might reveal a first-corner frequency that reflects overall rupture length (e.g., static stress drop) but not a second-corner frequency related to the slip-pulse length.
Although the first prosaic explanation cannot be ruled out, I will explore the second explanation. If the results presented in this article do reflect a nonsimilar rupture process (i.e., a slip-pulse model), then one would infer that the slippulse length is not strongly dependent on moment over the magnitude range 4.5-6. Because the $M 4-5$ events in this study have expected source radii on the order of $\approx 1.5-4 \mathrm{~km}$, a common slip-pulse length would have to be smaller than these dimensions, perhaps on the order of $0.2-1 \mathrm{~km}$ (as an estimate). This suggests a slip-pulse length somewhat smaller than, but not inconsistent with, previous results for moderate earthquakes (Heaton, 1990).

It does seem implausible that the slip-pulse width would be completely constant; previous studies yield estimated slip-pulse lengths on the order of $0.8-2.7 \mathrm{~km}$ for several recent $M$ 6.9-7.3 events but of $13 \mathrm{~km}$ for the 19 September 1985 M 8.1 Michoacan, Mexico earthquake (Heaton, 1990) and approximately $10 \mathrm{~km}$ for the 28 June 1992 M 7.3 Landers, California earthquake (Wald and Heaton, 1994). Furthermore, it is also not tenable to suggest a nearly constant slip pulse for events much smaller than $M 4$, the overall dimensions of which will be limited.

A slip-pulse rupture model could also account for the subtle but systematic misfit of the Yucca Valley spectra evidenced in Figure 5 (and the relatively high mainshock Brune stress drop). That is, in order to account for an intermediate slope, the regression fits the highest spectral levels, which are flatter than they should be, at the expense of fitting the low-frequency levels. In this case, site response is considerably less well constrained, and the inferred site response will affect the frequency band over which the intermediate spectral slopes are inferred. This illustrates the fundamental difficulty in resolving site, path, and source effects, especially in a region for which independent site-response results are not available. However, an explanation (for the spectral characteristics) in terms of the source effect discussed previously is certainly plausible.

An examination of the spectra in Figure 5 suggests that an intermediate spectral slope generally biases the cornerfrequency regression results to somewhat higher-thanappropriate values. These results illustrate how the spurious scaling of stress drop with moment might result from shoehorning observed spectra into an inappropriate single-corner model, such as in a joint-inversion analysis, especially for earthquakes of magnitude 4 and larger. This could explain a systematic tendency for moderate events to be characterized by higher stress drop than small $(M<4)$ events, in either joint-inversion studies or EGF studies that cover a magnitude range of roughly $M$ 3-5 (e.g., Fletcher and Boatwright, 1991; Hough and Dreger, 1995). That is, if only the largest events in the dataset were associated with an intermediate spectral decay, then an apparent magnitude dependence of stress drop could result. These arguments are also consistent with the conclusions of Atkinson and Silva (1997), who infer an intermediate spectral decay from a large study of moderate-to-large California earthquakes. It appears 
that fitting a one-corner model with an exponential decay to a spectrum with a broad intermediate slope results in significant biases in the estimation of corner frequencies.

\section{Conclusions}

The two datasets presented in this study are rather small. Unfortunately, good event clusters with a mainshock magnitude of $M 5.5+$ are limited by a number of disparate factors: (1) the small number of larger events during the few years that broadband networks have been in existence, (2) an apparent tendency for the largest earthquakes to not generate good EGF events, and (3) instrumental difficulties in obtaining on-scale recordings of large events and their early aftershocks (the latter often providing the best candidate EGF events (Hough, 1997).

Analyzing the best data available from recent earthquake sequences in California, I find that, while mainshocksource spectral shapes obtained using EGF analysis are apparently consistent with an $\omega^{2}$ source, the observed spectra reveal an intermediate decay that is less steep than $\omega^{2}$. These latter results are consistent in some respects with the model presented by Haddon (1996) that incorporates directivity and a fractional stress-drop rupture. Finite-fault models can also lead to an intermediate spectral decay (Beresnev and Atkinson, 1999). However, these models predict that the intermediate slopes would also be observed on source spectra obtained with EGF deconvolutions. The contradictory results obtained in this article could reflect either of the bandwidth limitations. They could also reflect a fractional stress-drop model with a rupture process that is not self-similar, such as a slip-pulse model in which the slip pulse controls the radiated energy and has a length not strongly dependent on seismic moment.

The conclusions are also generally consistent with a number of disparate studies of high-frequency ground motions (e.g., Hanks and Boore, 1984), particularly the results that ground motions for $M>4$ earthquakes at frequencies above their corners are typically characterized by stress-drop values higher than the static stress-drop values typically inferred for moderate-to-large earthquakes.

The behavior of $\sigma_{\mathrm{b}}$ itself is expected to be complicated because of biases introduced by application of an inappropriate one-corner model for larger events. Brune stressdrop, $\sigma_{\mathrm{b}}$, and static stress drop may be consistent for $M<5$ events, so compilations that combine $\sigma_{\mathrm{b}}$ for smaller events with static stress drop for larger events (e.g., Hough, 1996) may appear relatively seamless and constant with moment, possibly with a slight bump at moderate magnitudes.

The only other obvious explanation for the apparent versus static stress-drop paradox requires that estimates of radiated energy/apparent stress be universally biased by an incomplete accounting for site response or surface-wave energy. In addition to the difficulties, discussed above, of explaining the PFO observations in terms of site response, this seems implausible for several reasons: (1) high apparent stress drops are found for Terrascope stations representing a wide range of near-site geology, and (2) biases caused by site response would not explain the observed systematics of apparent stress-drop scaling.

The nearly constant slip-pulse model discussed in this article is not, at first glance, consistent with the conclusions of Frankel (1995) who simulates strong-motion recordings of the $M 6.91989$ Loma Prieta mainshock using $M$ 3.7-4 aftershocks as EGF events and concludes that the mainshock spectral shapes are well matched using a summation scheme based on constant static stress drop. Because a $M 3.8$ event with a $4 \mathrm{MPa}$ stress drop will have an overall rupture dimension of roughly $800 \mathrm{~m}$, it is unlikely to have a slip-pulse extent comparable to the mainshock, so one would not expect the spectral summation to match that of the mainshock. However, close inspection of figure 16 of Frankel (1995) reveals that, at four of the five sites analyzed, the simulated spectral amplitudes underpredict the observed mainshock amplitudes by approximately a factor of 2 , between $0.5 \mathrm{~Hz}$ (the lowest frequency analyzed) and roughly $3 \mathrm{~Hz}$. While it is arguable whether this misfit is significant, the character of the residuals is at least consistent with the mainshock spectra having an intermediate slope within this frequency range that is not present in the EGF spectra.

The subtle nature of the (possible) misfit in the Frankel (1995) results is echoed in the results presented in this article in Figure 3b. A consideration of the high- versus lowfrequency deconvolved amplitudes suggests that the inferred intermediate spectral regime does not strongly affect the high-frequency scaling. If this is the case, then it is reasonable that the intermediate spectral decay would be best revealed in energy calculations (that integrate over the entire bandwidth) rather than calculations based on high-frequency spectral levels or scaling.

The association of an intermediate spectral decay with a slip-pulse model is not new; previous studies (e.g., Mayeda and Walter, 1996) have pointed out that it may be quite misleading to base predicted earthquake ground motion (and/or spectral characteristics) on a simple one-corner model with a constant static stress drop. Although this model may characterize part of the earthquake rupture process (the propagation of the slip pulse along the full extent of the rupture), it will not fully characterize the radiated seismic energy.

By examining both the deconvolved source spectra estimated using the EGF method and the raw spectra, this study reveals that each method appears to reveal a distinct aspect of the earthquake rupture process: simple, $\omega^{2}$, spectral shapes and a self-similar static stress drop on one hand and complex spectral shapes and a nonconstant apparent stress drop on the other. I conclude that the combined observations, from this study and many others, suggest that radiated spectra could be significantly shaped by directivity and/or effects related to the propagation of ruptures as a narrow slip pulse. 


\section{Acknowledgments}

I thank Hiroo Kanamori, Tom Heaton, Doug Dreger, Tom Hanks, Bill Joyner, Gail Atkinson, Nancy King, and Art Frankel for helpful discussions in the course of this work and Katrin Hafner for assistance with processing the codes for the Terrascope data. I thank Yoshihas Iio and two anonymous reviewers for helpful comments and very gratefully acknowledge Michael Fehler for his suggestions and patience. The Joshua Tree/Landers GEOS deployment involved the hard work of numerous individuals, including Jim Mori, Gene Sembera, Chris Dietel, Joe Sena, Gary Glassmoyer, and Scott Lydeen, and the helpful cooperation of Ralph Skaggs and Ginny Whitney. The Terrascope data was accessed through the Southern California Earthquake Center data center, which is supported by grants from the National Science Foundation and the United States Geological Survey.

\section{References}

Abercrombie, R., and P. C. Leary (1993). Source parameters of small earthquakes recorded at $2.5 \mathrm{~km}$ depth, Cajon Pass, California: implications for earthquake scaling, Geophys. Res. Lett. 20, 1511-1514.

Aki, K. (1991). Summary of discussions on coda waves at the Istanbul IASPEI meeting, Phys. Earth Planet. Interiors 67, 1-3.

Anderson, J. G., and S. E. Hough (1984). A model for the shape of the Fourier amplitude spectrum of acceleration at high frequencies, Bull. Seism. Soc. Am. 74, 1969-1994.

Aster, R. C., and P. M. Shearer (1991). High-frequency borehole seismograms recorded in the San Jacinto fault zone, southern California. II. Attenuation and site effects, Bull. Seism. Soc. Am. 81, 1081-1101.

Atkinson, G. M., and W. Silva (1997). An empirical study of earthquake source spectra for California earthquakes, Bull. Seism. Soc. Am. 87, $97-113$.

Beresnev, I. A., and G. M. Atkinson (1999). Generic finite-fault model for ground-motion prediction in eastern North America, Bull. Seism. Soc. Am. 89, 608-625.

Berger, J., L. M. Baker, J. N. Brune, J. B. Fletcher, T. C. Hanks, and F. L. Vernon (1984). The Anza array: a high-dynamic range, broadband, digitally radiotelemetered seismic array, Bull. Seism. Soc. Am. 74, $1469-1481$.

Boatwright, J., and G. L. Choy (1992). Acceleration source spectra anticipated for large earthquakes in northeastern North America, Bull. Seism. Soc. Am. 82, 660-682.

Borcherdt, R. G., J. B. Fletcher, E. G. Jensen, L. Maxwell, J. R. Van Shaak, R. E. Warrick, E. Cranswick, J. S. Johnston, and R. McClearn (1985). A general earthquake-observation system, Bull. Seism. Soc. Am. 75, $1783-1825$.

Brune, J. N. (1970). Tectonic stress and the seismic shear waves from earthquakes, J. Geophys. Res. 75, 4997-5009.

Brune, J. N., R. J. Archuleta, and S. Hartzell (1979). Far-field S-wave spectra, corner frequencies, and pulse shapes, J. Geophys. Res. 84, 22622272.

Dreger, D. S. (1994). Empirical Green's function study of the January 17, 1994 Northridge, California earthquake, Geophys. Res. Lett. 21, 2633-2636.

Fletcher, J. B., T. Fumal, H.-P. Liu, and L. C. Haar (1990). Near-surface velocities and attenuation at two boreholes near Anza, California, from logging data, Bull. Seism. Soc. Am. 80, 807-831.

Fletcher, J. B., and J. Boatwright (1991). Source parameters of Loma Prieta aftershocks and wave propagation characteristics along the San Francisco Pennisula from a joint inversion of digital seismograms, Bull. Seism. Soc. Am. 81, 1783-1812.

Frankel, A. (1991). High frequency spectral falloff of earthquakes, fractal dimension of complex rupture, b-value, and the scaling of stress on faults, J. Geophys. Res. 96, 6291-6302.

Frankel, A. (1995). Simulating strong motions of large earthquakes using recordings of small earthquakes: the Loma Prieta mainshock as a test case, Bull. Seism. Soc. Am. 85, 1144-1160.
Frankel, A., J. Fletcher, F. Vernon, L. Haar, J. Berger, T. Hanks, and J. Brune (1986). Rupture characteristics and tomographic source imaging of $M_{l} \approx 3$ earthquakes near Anza, southern California, J. Geophys. Res. 91, 12633-12650.

Haddon, R. A. W. (1996). Earthquake source spectra in eastern North America, Bull. Seism. Soc. Am. 86, 1300-1313.

Hanks, T. C. (1979). b values and $\omega^{-\gamma}$ seismic source models: implications for tectonic stress variations along active crustal fault zones and the estimation of high-frequency strong ground motion, J. Geophys. Res. 84, 2235-2242.

Hanks, T. C., and D. M. Boore (1984). Moment-magnitude relations in theory and practice, J. Geophys. Res. 89, 6229-6235.

Haskell, N. A. (1964). Total energy and energy spectral density of elastic wave propagation from propagating faults, Bull. Seism. Soc. Am. 54, 1811-1841.

Hauksson, E., L. M. Jones, K. Hutton, and D. Eberhart-Phillips (1993). The 1992 Landers earthquake sequence-seismological observations, $J$. Geophys. Res. 98, 19835-19858.

Hauksson, E., K. Hutton, H. Kanamori, L. Jones, J. Mori, S. Hough, and G. Roquemore (1995). Preliminary report on the 1995 Ridgecrest earthquake sequence in eastern California, Seism. Res. Lett. 66, $54-60$.

Heaton, T. H. (1990). Evidence for and implications of self-healing pulses of slip in earthquake rupture, Phys. Earth Planet. Interiors 64, 1-20.

Hough, S. E. (1994). Southern surface rupture associated with the 1992 Landers earthquake, Bull. Seism. Soc. Am. 84.

Hough, S. E. (1996). Observational constraints on earthquake source scaling: understanding the limits in resolution, Tectonophysics 261, 83-96.

Hough, S. E. (1997). Empirical Green's function analysis: taking the next step, J. Geophys. Res. 102.

Hough, S. E., and J. G. Anderson (1988). High-frequency spectra observed at Anza, California: implications for Q structure, Bull. Seism. Soc. Am. 78, 692-707.

Hough, S. E., J. Mori, E. Sembera, G. Glassmoyer, C. Mueller, and S. Lydeen (1993). Southern surface rupture associated with the $1992 \mathrm{M}$ 7.4 Landers earthquake: did it all happen during the mainshock?, Geophys. Res. Lett. 20, 2615-2618.

Hough, S. E., and D. S. Dreger (1995). Source parameters of the 4/23/92 Joshua Tree, California, earthquake and its aftershocks: empirical Green's function analysis of TERRAscope and GEOS data, Bull. Seism. Soc. Am. 85, 1576-1590.

Hough, S. E., J. Lees, and F. Monastero (1999). Attenuation and source properties at the Coso geothermal region, California, Bull. Seism. Soc. Am. 89, 1606-1619.

Humphrey, J. R., and J. G. Anderson (1995). Seismic source parameters from the Guerrero subduction zone, Bull. Seism. Soc. Am. 84, 1754 1769.

Jones, L. M., K. Aki, D. Boore, M. Celebi, A. Donnellan, J. Hall, R. Harris, E. Hauksson, T. Heaton, et al. (1994). The magnitude-6.7 Northridge, California earthquake of 17 January 1994, Science 266, 389-397.

Jones, L. E., and S. E. Hough (1995). Analysis of broadband recordings of the 6/28/92 Big Bear Earthquake: evidence for a multiple-event source, Bull. Seism. Soc. Am. 85, 688-704.

Kanamori, H., E. Hauksson, and T. Heaton (1991). TERRAscope and CUBE project at Caltech, EOS 72, 564.

Kanamori, H., J. Mori, E. Hauksson, T. H. Heaton, L. K. Hutton, and L. M. Jones (1993). Determination of earthquake energy release and ML using TERRAscope, Bull. Seism. Soc. Am. 83, 330-346.

Kato, K., K. Aki, and M. Takemura (1995). Site amplification from coda waves: validation and application to S-wave site response, Bull. Seism. Soc. Am. 85, 467-477.

Kennett, B. L. N., and N. J. Kerry (1979). Seismic waves in a stratified half space, Geophys. J. R. Astron. Soc. 57, 557-583.

Mayeda, K., and W. R. Walter (1996). Moment, energy, stress drop, and source spectra of western United States earthquakes from regional coda waves, J. Geophys. Res. 101, 11,195-11,208. 
Mori, J., and A. Frankel (1990). Source parameters for small events associated with the 1986 North Palm Springs, California, earthquake determined using the empirical Green's function method, Bull. Seism. Soc. Am. 80, 278-295.

Raoof, M., R. B. Herrmann, and L. Malagnini (1999). Attenuation and excitation of three-component ground motion in southern California, Bull. Seism. Soc. Am. 89, 888-902.

Scott, J. S., T. G. Masters, and F. L. Vernon (1994). 3-d velocity structure of the San Jacinto fault zone near Anza, California. I. P-waves, Geophys. J. Int. 119, 611-626.

Singh, S., and R. B. Herrmann (1983). Regionalization of crustal coda-Q in the continental United States, J. Geophys. Res. 88, 527-538.

Smith, K. D., J. N. Brune, and K. F. Priestley (1991). The seismic spectrum, radiated energy, and the Savage and Wood inequality for complex earthquakes, Tectonophysics 188, 303-320.

Velasco, A. A., C. J. Ammon, and T. Lay (1994). Empirical Green-function deconvolution of broad-band surface-waves-rupture directivity of the 1992 Landers, California $(\mathrm{Mw}=7.3)$ earthquake, Bull. Seism. Soc. Am. 84, 735-750.

Vernon, F. L. (1989). Analysis of data recorded on the ANZA seismic network, Ph.D. Thesis, University of California, San Diego, California.

Wald, D. J., and T. H. Heaton (1994). Spatial and temporal distribution of slip for the 1992 Landers, California, Earthquake, Bull. Seism. Soc. Am. 84, 668-691.

Walter, W. R., J. N. Brune, and K. Priestley (1988). Observations of high frequency P-wave earthquake and explosion spectra compared with $\omega^{-3}, \omega^{-2}$, and Sharpe source models, J. Geophys. Res. 93, 63186324.

United States Geological Survey

Pasadena, California

(S.E.H.)

Manuscript received 24 September 1997. 